\title{
Ist der Oberharz im Pleistozän vergletschert gewesen?
}

\author{
Von Klaus DupHorn, Hannover
}

Mit 4 Abbildungen

$\mathrm{Zusammenfassung.} \mathrm{Einige} \mathrm{Ergebnisse} \mathrm{zur} \mathrm{Frage} \mathrm{der} \mathrm{pleistozänen} \mathrm{Harzvergletsche-}$ rungen werden mitgeteilt. Diese Ergebnisse, die bei einer geologischen Kartierung des oberen Odertales gewonnen wurden, erbringen den Nachweis, daß dieses Gebiet im Pleistozän vergletschert war.

Die Geländeuntersuchungen im Odertal konzentrierten sich auf vier Endmoränenbögen und die mit ihnen verknüpften Ablagerungen, die in folgende geologisch-geomorphologische Einheiten untergliedert werden können: 1) Sander-Vorfeld, 2) Endmoränenlandschaft mit Blockwällen, Schmelzwasserrinnen und Toteislöchern, 3) Seitenmoränenlandschaft mit Kamesterrassen, Schmelzwasserrinnen, Toteislöchern, paraglazialen Teilfeldern und erratischen Geschieben, 4) Gletscherzungenbecken mit bis zu $23 \mathrm{~m}$ mächtigen, glazilimnischen Sedimenten.

$\mathrm{Su} \mathrm{m} \mathrm{m}$ a r y. Thip paper deals with results concerning the problem of Pleistocene glaciation in the Harz Mountains. These results obtained by geological mapping in the upper Oder Valley proove the existence of a local glacier in the Pleistocene.

Primarily the field work in the Oder Valley was concentrate to four end moraine lobes and to the deposits connected with them. This area can be subdivided into the following geological/ morphological units: 1) Proglacial outwash plain, 2) end moraine landscape with boulder walls, channels of meltwater and dead ice kettles, 3) lateral moraine landscape with kames, channels of meltwater, dead ice kettles, paraglacial fields and erratic boulders, 4) basin of glacier tongue filled with glaciolimnic deposits up till $23 \mathrm{~m}$ thick.

\section{Einleitung}

„Steine sind stumme Lehrer. Sie machen den Beobachter stumm und das Beste, was man von ihnen lernt, ist, nichts mitzuteilen" (J. W. v. Goethe).

Offenbar gibt es auf der Erde nicht mehr genug Steine, denn die Flut der geowissenschaftlichen Publikationen steigt immer höher; sie kann heute, da mit einem jährlichen Ausstoß von ca. 40000 Veröffentlichungen gerechnet wird, selbst von den kühnsten Wellenreitern nicht mehr überblickt werden. Das gilt sogar schon für die Spezialbereiche kleinerer Dimensionen, z. B. auch für die jüngste und mit Abstand kürzeste Formation der Erdgeschichte, das Quartär.

Wenn ich hiermit trotzdem zur Feder greife und der Vielzahl der (von anderen Autoren) schon geschriebenen quartärgeologischen Publikationen noch eine weitere hinzufüge, so geschieht das, um dem Jubilar, dem diese Festschrift gewidmet ist und mit dem ich seit mehreren Jahren in der Redaktionskommission für die Internationale Quartärkarte von Europa zusammenarbeite, auf diesem Wege für die vielen Anregungen, die ich dabei von ihm empfangen habe, meinen Dank abzustatten. Das glaube ich am besten tun zu können, indem ich sein persönliches Jubiläum mit einem quartärgeologischen verbinde, dem auch er sich verbunden fühlen wird.

Dieses zweite Jubiläum betrifft die Erforschung der quartären Geschichte des Harzes. Es sind nämlich heuer genau hundert Jahre her, seit die Diskussion über die pleistozäne Eigenvergletscherung dieses Gebirges eröffnet wurde. Trotz der Zeitspanne von einem vollen Jahrhundert ist diese Diskussion bis zur Gegenwart in Gang geblieben. Seit K. H. ZimmermanN (1868) erstmals „über Gletscherspuren im Harz" berichtete, stehen sich noch immer Argumente und Gegenargumente für bzw. wider die pleistozäne Eigenvergletscherung des Harzes gegenüber und noch immer reicht die Spanne der Meinungen von umfangreichen Plateau- und Talvergletscherungen bis zur gänzlichen Ablehnung selbst von schwachen und lokal begrenzten Eisbewegungen. 
Es ist hier nicht der Platz, um einen ausführlichen Abriß über die Erforschung der quartären Deckschichten des Harzes zu geben. Es sei jedoch in dem hier gesteckten Rahmen erwähnt, daß sich auch P. Woldstedt (1935), wie die meisten Geologen, die dem Problem der pleistozänen Eigenvergletscherung des Harzes nachgingen, auf die Seite der Glazialisten gestellt hat, daß aber dennoch in den letzten Jahren die vornehmlich von Geographen geäußerten, antiglazialen Argumente die Oberhand gewannen. So hält H. Lembke (1961) die zuletzt von H. Poser \& J. Hövermann (1951) als weichseleiszeitliche Moränen gedeuteten, ungeschichteten Talschuttmassen im Westharz für Solifluktionsschutt und Wildbachschotter, die talabwärts in die Obere Niederterrasse übergehen. Auch für den nördlichen und östlichen Teil des Brockenmassivs wird eine Vergletscherung abgelehnt (E. Mücke, 1964; G. Reichelt, 1964). Die antiglazialen Deutungen, die mit den paläoklimatischen Erwägungen von J. BüDEL (1960) über den Klimagang der WeichselKaltzeit, wonach die klimatische Schneegrenze im Harz während des Hochglazials in ca. $1000 \mathrm{~m}$ Höhe ü.N.N. gelegen haben soll, gut übereinstimmen, haben trotz der Ergebnisse von H. Poser \& J. Hövermann (1951), nach denen die Annahme einer tief in die Täler des Westharzes hinabreichenden, weichseleiszeitlichen Vergletscherung gut begründet zu sein schien, auch in das neueste Lehrbuch über die Geologie des Harzes (G. Möвus, 1966) Eingang gefunden.

Den o. g. Diskrepanzen liegen zwei Hauptursachen zugrunde:

1. beruht keine der vielen Interpretationen auf einer gezielt angesetzten, quartärgeologischen Spezialkartierung und

2. sind die meisten Autoren von einer falschen Grundauffassung, nämlich vom Denkmodell eines alpinen Formenschatzes, ausgegangen.

Im folgenden werden die ersten Ergebnisse einer quartärgeologischen Spezialkartierung im Maßstab 1:5000 mitgeteilt. Die Kartierung wurde im oberen Odertal durchgeführt, in einem Talabschnitt, der schon seit langem im Mittelpunkt der Diskussionen über das Problem der pleistozänen Eigenvergletscherung des Harzes steht. Weitere Untersuchungen, auch in den benachbarten Tälern, sind im Gange. Nach Abschluß der Untersuchung ist eine ausführliche Beschreibung der glaziären Ablagerungen und Formen im Oberharz vorgesehen.

Wenn nach all den widersprüchlichen Auffassungen, die im Verlaufe eines Jahrhunderts von vielen Autoren geäußert worden waren, überhaupt noch Hoffnung bestand, definitive Beweise für oder gegen die pleistozäne Eigenvergletscherung des Gebirges zu finden, dann mußten die Untersuchungen im Odertal angesetzt werden. Intensive geologisch-geomorphologische Kleinarbeit an dieser höffigsten Stelle mußte zunächst vor extensiverer Überschau den Vorrang erhalten.

\section{Morphologisch-geologische Ưbersicht}

Das Odertal ist das größte Tal der Südabdachung des Harzes. Das bis zum Staudamm des Oderteiches (ca. $720 \mathrm{~m}$ ü.N.N.) sehr steil und tief eingeschnittene Tal geht dort talaufwärts in ein weitspanniges, sanftgeböschtes, muldenförmiges Hochtal über, das eine Fläche von über $12 \mathrm{~km}^{2}$ einnimmt und dessen Umrandung im Osten mit dem Achtermann und im Westen mit dem Bruchberg auf Höhen von über $900 \mathrm{~m}$ ü. N. N. ansteigt.

Dieses stark vermoorte Hochtal, das bei einer entsprechenden Depression der klimatischen Schneegrenze auf Grund seiner orographischen Gestaltung für eine Firnakkumulation geradezu prädestiniert ist, liegt überwiegend im Brockengranit. Neben dem normalen Kerngranit kommen hier auch Varietäten der Rand- und Dachfazies vor. ${ }^{1}$ ) Im

1) Die Kenntnis der Varietäten des Brockengranits und ihrer Verbreitung ist m. E. für die Beurteilung der umstrittenen Frage der erratischen Geschiebe im Oberharz unerläßlich. 


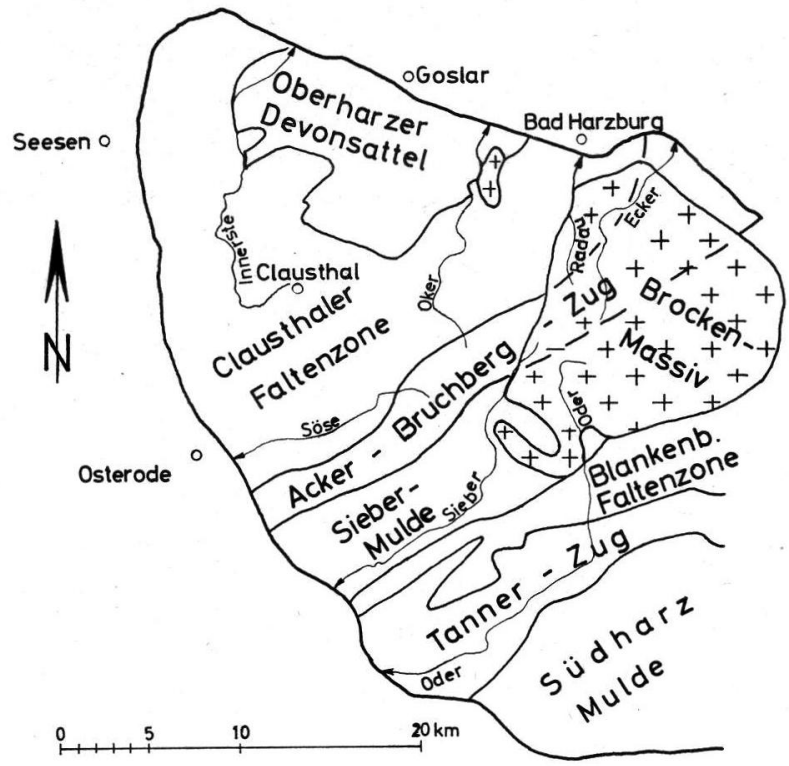

Abb. 1. Die geologischen Einheiten des Westharzes (zusammengestellt nach der Geologischen Úbersichtskarte von Nordwestdeutschland 1:300 000).

NW grenzt der Granit an die vorherrschend quarzitischen, oberdevonischen bis unterkarbonischen Acker-Bruchberg-Schichten. Vom Hahnenklee talabwärts streichen am östlichen Talhang hauptsächlich Tonschiefer-, Grauwacken- und Kalksilikathornfelse des Unterkarbons und Devons aus, während am westlichen Talhang bis zur Einmüdung des Kellwassers noch Granite vorherrschen (Abb. 1 u. 2).

\section{Kartierkonzeption}

Die Talsohle des oberen Odertales wird von blockreichen Schuttmassen eingenommen. Zwischen dem Rehberg im Westen und dem Hahnenklee im Osten verfüllen diese Schuttmassen das Tal in einer Mächtigkeit bis $30 \mathrm{~m}$ so mit grobem Blockwerk, daß der Fluß sich nur in einer engen Kerbe hindurchwinden kann. Hier befindet sich die einzigste Stelle im Harz, an der die Talschuttmassen in morphologisch markante Blockwälle und z. T. abflußlose Hohlformen von rinnen- und kesselförmiger Gestalt gegliedert sind.

Die Hauptmasse der hier beschriebenen Schuttmassen im Odertal, ca. $4 \mathrm{~km}$ nordöstlich von St. Andreasberg, zwischen 530 und $580 \mathrm{~m}$ ü. N.N., ist zweifellos in talabwärtiger Richtung und nicht durch seitliche Materialzufuhr von den Talhängen her antransportiert worden. Die Schuttkomponenten bestehen z. T. aus Gesteinen, die, wie z. B. der AckerBruchberg-Quarzit, nur im obersten Einzugsgebiet des Flusses anstehen (Abb. 1). Für die lithogenetische Deutung verblieb nur eine Alternative: Moränen oder talabwärts geflossene, periglaziäre Solifluktionsströme und Wildbachschotter. Die jüngsten Exponenten der beiden Richtungen wurden bereits genannt.

Gesetzt den Fall, die vier markanten Blockwälle, die - lobenförmig talabwärts weisend - das Odertal queren und stellenweise sowohl talauf- als auch talabwärts ihre Umgebung deutlich um mehrere Meter überragen (Abb. 2), sind Endmoränen eines Talgletschers, dann liegt es nahe anzunehmen, daß die bis $30 \mathrm{~m}$ über die Talsohle reichenden Wälle das Tal in der nachfolgenden Abschmelzperiode zeitweilig plombierten. Möglicherweise wurde während dieser Zeit dahinter sogar ein Stausee abgedämmt, in den die 
Schmelzwässer des abtauenden Gletschers Sedimente einschwemmten. Allein der Nachweis solch einer Verknüpfung der problematischen Schuttmassen mit glazilimnischen Staubeckensedimenten würde genügen, um eine Deutung als Wildbáchschotter oder Solifluktionsschutt auszuschließen. Auf Grund dieser Überlegungen galt dem Talabschnitt unmittelbar hinter den Blockwällen, der von den bisherigen Bearbeitern mit Ausnahme von A. BoDE (1908) gänzlich vernachlässigt worden war, besondere Aufmerksamkeit.

Bei glaziärer Akkumulation der Blockwälle im Zuge kleinerer Gletscheroszillationen bei im großen und ganzen stationärer Lage der Gletscherzunge sollten sowohl in den Hohlformen zwischen den Wällen (Abb. 2) als auch im Vorfeld Reste von glazilimnischen bis glazifluviatilen Sedimenten zu finden sein. Namentlich in den zahlreichen abflußlosen Senken dürften dann mächtigere Sedimentfüllungen vermutet werden.

Die oben geschilderten Aspekte zwangen zu Aufschürfungen und Peilstangenbohrungen. Dabei wurde die theoretische Konzeption bestätigt. Um noch tiefer in die hinter, zwischen und vor den Blockwällen gefundenen Sedimente eindringen zu können, wurde nun ein Drillbohrgerät zuhilfegenommen. Die Kartierung stützt sich wesentlich auf ein dichtes Netz von Peilstangen- und Drillbohrungen. Allein mit dem Drillbohrgerät, das auf einem Unimog montiert ist und mit einer rotierenden Schnecke von $8 \mathrm{~cm}$ Durchmesser arbeitet, wurden im hier beschriebenen Kartiergebiet über 80 Bohrungen bis zu einer Teufe von $15 \mathrm{~m}$ niedergebracht.

Dem einen oder anderen Leser, der mit der quartären Materie im Harz weniger vertraut ist, mag der auf kleiner Fläche betriebene technische Aufwand ziemlich groß vorkommen. Tatsächlich kann der Kartierer sich solch einen Aufwand nur in Ausnahmefällen leisten. So ein Ausnahmefall lag hier vor. Es kann nicht genug betont werden, daß Grundlagenforschung im engsten Sinne des Wortes betrieben werden mußte, weil die Diskussion über das Problem der pleistozänen Eigenvergletscherung des Harzes nach einem jahrhundertlangen Wirrwarr von Meinungen so festgefahren war, daß bei dieser gegebenen Situation neue Ergebnisse wirklich hieb- und stichfest sein mußten, um einerseits der zu erwartenden Kritik standhalten und andererseits für die weitere Erforschung der quartären Deckschichten in diesem Gebirge neue Impulse auslösen zu können.

\section{Kartierergebnisse}

Der Moränencharakter der o.g. und in der Abb. 2 dargestellten Schuttmassen im Odertal konnte durch eine quartärgeologische Spezialkartierung im Maßstab 1:5000 unter besonderer Berücksichtigung der geomorphologischen Formen und des paläozoischen Untergrundes (Neukartierung der Granitvarietäten und der Grenze Granit/Hornfels im obersten Talabschnitt) nachgewiesen werden. Die Ergebnisse der geologischen Kartierung und der geomorphologischen Formenanalyse stimmten dabei an vielen Stellen geradezu lehrbuchhaft überein.

Es gibt im Odertal mehrere Moränenstaffeln. Im folgenden werden nur die geologischmorphogenetischen Einheiten der markantesten Moränenstaffel unterhalb der Hahnenkleeklippen beschrieben. Diese Moränenstaffel gliedert sich in vier solcher Einheiten:

1. Sander-Vorfeld,

2. Endmoränenlandschaft mit Blockwällen, Schmelzwasserrinnen und Toteislöchern,

3. Seitenmoränenlandschaft mit Schmelzwasserrinnen, Kames-Terrassen, paraglazialen Teilfeldern und erratischen Geschieben,

4. Gletscherzungenbecken mit glazilimnischen Staubeckensedimenten.

1. Das Sander-Vorfeld: Der äußersten Moränenstufe ist eine sanft und gleichmäßige talabwärts geneigte $\left(3-5^{\circ}\right)$, nur mäßig reliefierte Schwemmfläche vorgelagert. Diese Fläche erstreckt sich vom Hangfuß des Hahnenklee über die ganze Talsohle bis zur Oder. 


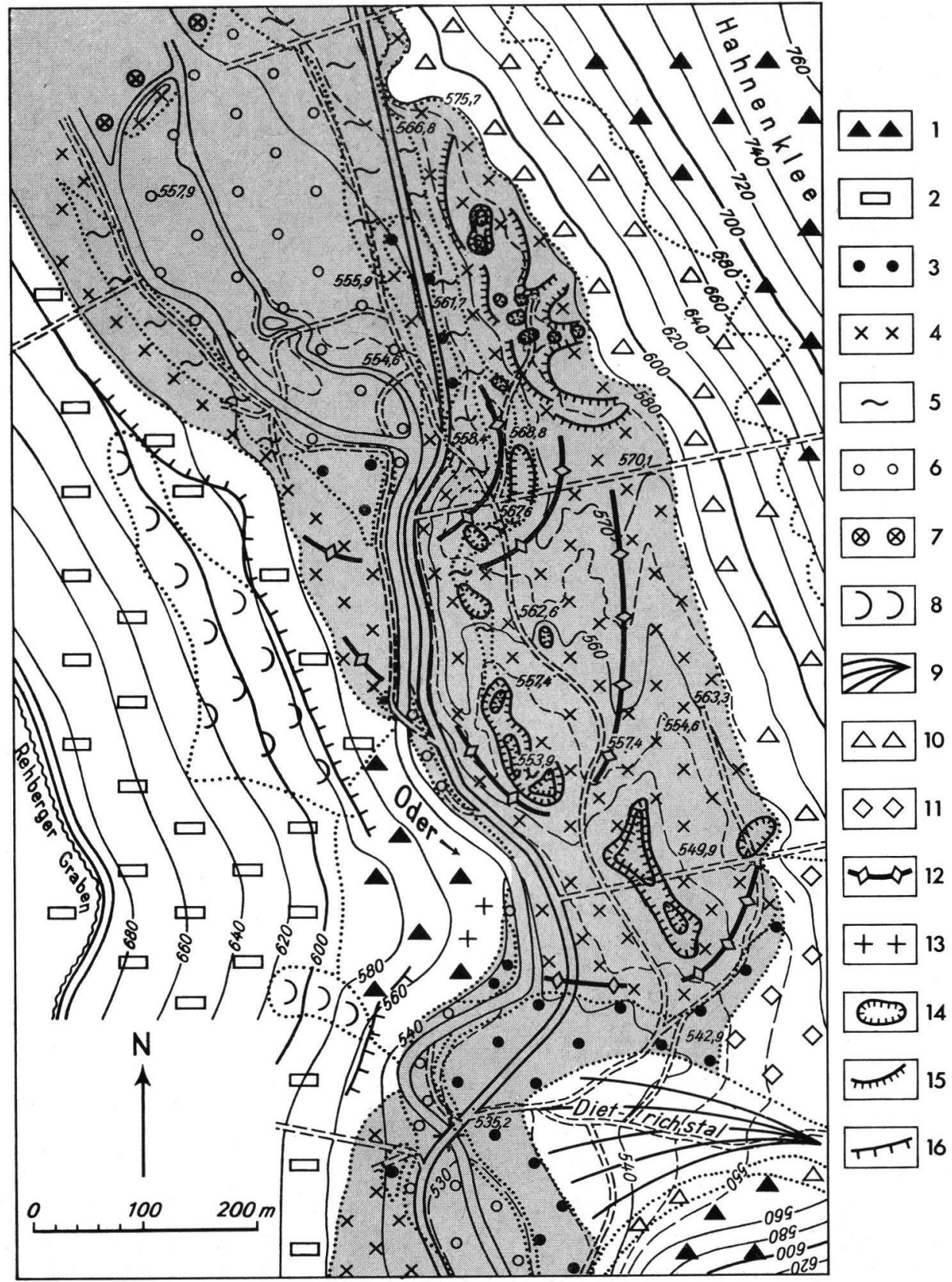

Abb. 2. Geologisch-geomorphologische Spezialkarte 1:5000 des oberen Odertales zwischen dem Hahnenklee und dem Rehberg nordöstlich von St. Andreasberg.

Legende zu Abb. 2: 1 = Grauwacken- und Tonschieferhornfels, Unterkarbon, 2 = porphyrartiger Granit, Oberkarbon, $3=$ glazifluviatile Kiese, Weichselkaltzeit, $4=$ Moräne, Weichselkaltzeit, 5 = Glazilimnische Ablagerungen, meist Staubeckenschluff, Weichselkaltzeit, 6 = periglaziär-fluviatile, blockreiche Kiese der Unteren Niederterrasse, Weichselkaltzeit, $7=$ periglaziärfluviatil überprägte Moräne, Weichselkaltzeit, $8=$ Hangsolifluktionsschutt, Weichselkaltzeit, 9 = Schuttschwemmfächer, Weichselkaltzeit bis Holozän, $10=$ Holozäner Hangschutt aus unterkarbonischen Grauwacken- und Tonschieferhornfelsen, $11=$ Holozäner Hangschutt aus devonischen Kalksilikat- und Tonschieferhornfelsen, $12=$ Endmoränen, $13=$ erratische Geschiebe: $14=$ Toteislöcher, $15=$ Kamesterrassen, $16=40-\mathrm{m}$-Terrasse. 
Talabwärts läuft sie unmittelbar südlich der Blattgrenze (Abb. 2) hinter der nächstälteren Moränenstaffel aus.

Die Sanderfläche wird von fließgeschichteten, sandigen Grobkiesen eingenommen. Die Matrix wird in Richtung zum Hahnenklee hin immer schluffiger. Der Geröllbestand ist derselbe wie im Blockschutt der Endmoränen: überwiegend Granite und Hornfelse, daneben Acker-Bruchberg-Quarzit sowie etwas Kieselschiefer und Gangquarz. Die Mächtigkeit der Sanderkiese ist ziemlich gering; durchschnittlich beträgt sie 1,5-2 m.

An der Einmündung des Dietrichstales werden die Schmelzwasserkiese von schluffigem Hornfelssplit überlagert, der einen morphologisch sehr deutlich ausgeprägten Schwemmfächer aufbaut. Die Mächtigkeit des Schuttschwemmfächers, dessen Hangendposition gegenüber den Sandersedimenten durch die Drillbohrungen belegt ist, beträgt ca. $4 \mathrm{~m}$.

An der Oder wird die Sanderfläche etwa $2 \mathrm{~m}$ tief von der Niederterrasse unterschnitten. An der Terrassenstufe kommt unter den Schmelzwasserkiesen schluffig-sandiger, grobblockiger Moränenschutt zutage, der die hier beschriebenen Blockschuttwälle mit den weiter talabwärts liegenden Moränenstaffeln verbindet.

Die Endmoräne sitzt der Sanderwurzel mit markanter Steilstufe auf. Daß die glazifluviatilen Ablagerungen sich unter die Endmoräne einschieben, geht aus Einschlüssen von Sandschollen, die im gefrorenen Zustand in die Moräne eingearbeitet wurden, hervor. Die sub- bzw. intramoränale Ausdehnung dieser glazifluviatilen Ablagerungen scheint jedoch beschränkt zu sein.

2. Die Endmoränenlandschaft ist morphologisch und geologisch stark gegliedert. Zunächst fällt die morphologische Gliederung in Voll- und Hohlformen ins Auge. Am auffälligsten sind die bereits genannten Blockwälle.

H. Lемвке (1961) leugnet das Vorhandensein der Blockwälle und gliedert den Schutt in drei talabwärts fallende Stufen, die er als Solifluktionsstufen deutet. H. Poser \& J. Hövermann (1951) weisen jedoch darauf hin, daß eine derartige Deutung als Solifluktionsform voraussetzt, daß Analoga dazu unter den Formen der rezenten Solifluktion nachweisbar sind. Dies ist aber in der hier vorliegenden Größenordnung nicht der Fall. Auch die neueste, sehr umfangreiche Literatur über rezente Solifluktionserscheinungen im subpolaren Klimabereich bietet keine analogen Beispiele.

Auf der topographischen Spezialkarte 1:5000, die im Auftrage der Königlich Preußischen geologischen Landesanstalt (A. BoDE, 1908) angefertigt worden war und die meiner Kartierung als Grundlage diente, tritt der Wallcharakter trotz der erosiven Zerschneidung der Endmoränenbögen deutlich hervor (Abb. 2). Nur der unterste Moränenbogen bildet eine Stufe. Die drei anderen Bögen, namentlich der zweite, die - von Erosionsrinnen durchbrochen - das Tal in seiner ganzen Breite queren, überragen ihre Umgebung sowohl talauf- als auch talabwärts um mehrere Meter.

Stellenweise scheinen die Blöcke, die vorherrschend aus kantengerundeten Graniten bestehen, wie von der Faust eines Riesen aufeinandergetürmt zu sein. Blöcke von $4 \mathrm{~m}$ Länge und 2,5 m Breite sind hier keine Seltenheit. Der größte Granitblock ist 4,8 m lang und $2,7 \mathrm{~m}$ breit.

Einen guten Einblick in das Gefüge der Moräne bietet der Einschnitt des Neumeierweges, der dort, wo der zweite Blockwall an die Odertalstraße stößt, von dieser Straße abzweigt und fortan die Oder entlang des westlichen Ufers flußaufwärts begleitet. Dieser Einschnitt ist so tief, daß darin die Grundmoräne zutagekommt.

Die Grundmoräne besteht aus einer ungeschichteten, graubraunen, schluffig-sandigen Grundmasse mit Geschieben bis zu 1,5 m Länge. Die Sandfraktion enthält hauptsächlich Granitgrus, Feldspäte und Quarz. Die Hauptmasse der Geschiebe, von denen viele steilgestellt auf schmaler Kante stehen, besteht aus fein- bis mittelkörnigen sowie aus porphyrischen Dachgraniten. Daneben kommen reichlich eckige bis kantengerundete Bruchstücke von Tonschiefer- und Grauwackenhornfelsen sowie glattgeschliffene Geschiebe von Acker- 
Bruchberg-Quarziten vor. Seltener sind Geschiebe von grobkörnigem Kerngranit und von mikropegmatitischem Randgranit aus dem obersten Talabschnitt. Kieselschiefer sind sehr selten.

Schon E. KAYSER (1892) hat in der Odertalmoräne gekritzte Hornfelsgeschiebe gefunden und als Beweis für den glaziären Charakter der Schuttmasse angeführt. Solche Schrammen, die es vereinzelt gibt, sind jedoch mehrdeutig. Weit mehr als sie weisen typische Facettengeschiebe von Acker-Bruchberg-Quarzit auf einen Ferntransport durch Gletschereis hin.

Zwischen den Moränenbögen liegen Hohlformen, die z. T. abflußlos sind, von rinnenund kesselförmiger Gestalt. Die Rinnen sind mit Schmelzwasserablagerungen - Kies, Sand und Schluff - angefüllt. Auf der Karte (Abb. 2) ist nur die Hauptrinne dargestellt, die an der Naht zwischen dem hintersten Moränenbogen und der am östlichen Talhang gelegenen Seitenmoräne ansetzt und sich über eine Reihe von Toteislöchern hinweg längs durch die gesamte Moränenstaffel erstreckt.

Die zahlreichen Toteislöcher in der Moränenlandschaft haben schon frühzeitig Aufmerksamkeit erregt. H. BoDE (1908) deutet sie als Auskolkungen von Schmelzwasserbächen. Als erste äußern H. Poser \& J. Hövermann (1951) den Verdacht, daß „d e n geschlossenen Hohlformen möglicherweise der Charakter von Toteislöchern zugeschrieben werden könnte" (S. 86). H. LembKe (1961) trägt zwar die größten dieser Hohlformen in eine Karte ein (S. 450), nimmt aber nicht $\mathrm{zu}$ deren Entstehung Stellung.

Daß es sich tatsächlich um Toteislöcher handelt, geht aus der Lage, der Form und der Sedimentfüllung hervor. Zwar sind, wie aus Abb. 2 ersichtlich ist, die Einsenkungen auch Leitbahnen für die abfließenden Schmelzwässer und - wie im folgenden noch erörtert wird - für überlaufendes Wasser aus dem hinter der Moränenstaffel abgedämmten Stausee gewesen; sie liegen jedoch nicht nur unmittelbar vor den Moränenwällen, wo sie durch herabfallende Sturzbäche ausgekolkt sein könnten, sondern auch dazwischen und dahinter, wo solch eine Auskolkung nicht möglich ist.

Auch die Form spricht gegen fluviatile bzw. glazifluviatile Erosion als Hauptagens. Die größte abflußlose Hohlform ist ca. $150 \mathrm{~m}$ lang und $20-40 \mathrm{~m}$ breit. Sie liegt hinter der untersten Moränenstufe. Ihre tiefste Stelle, die fast immer Wasser enthält, liegt unter dem Niveau der Kreuzung zwischen der die Einsenkung querenden Schneise und dem in Längsrichtung des Tales verlaufenden Waldwege. Das Niveau des Ausflusses liegt immer noch ca. $3 \mathrm{~m}$ über dem der ziemlich ebenen Oberfläche im Innern der Einsenkung.

Diese Einsenkung, deren Boden zuerst mit der Peilstange und dann mit dem Drillbohrgerät abgebohrt wurde, ist in sich nochmals in zwei Vertiefungen untergliedert. Sowohl die Wandungen der Großform als auch der flache Rücken zwischen den Vertiefungen innerhalb derselben bestehen aus grobblockigem Moränenschutt. In den Vertiefungen liegt gelbbrauner Schluff, der bis $6 \mathrm{~m}$ mächtig wird. Der Schluff ist sehr rein. Nur die basalen Portionen enthalten gelegentlich gröbere Komponenten bis hin zu größeren Geschieben.

Die hier beschriebene, abflußlose Hohlform steht über die o. g. Hauptrinne, in die weitere solcher Hohlformen eingelassen sind, mit dem Staubecken hinter der Moränenstaffel in Verbindung. Dieses Staubecken wurde beim weiteren Abschmelzen des Odertalgletschers von der Endmoränenstaffel abgedämmt. Im Zusammenhang mit der lithogenetischen Deutung der Sedimente in den Hohlformen der Moränenlandschaft sei hiermit vorweggenommen, daß bereits vor der Anlage des heutigen Oderlaufes auf der Westseite des Tales ein Überlauf des Stausees auf der Ostseite erfolgte, wobei die in den Stausee eingeschwemmte Gletschertrübe in die o. g. Rinnen und austauenden Toteislöcher weiterverfrachtet wurde. 
Daß die abflußlosen Hohlformen tatsächlich durch Toteis und nicht durch tiefenerosive Auskolkung entstanden, geht auch daraus hervor, daß die subcutanen Wandungen bis zu $60^{\circ}$ geneigt sind. Das sind Böschungswinkel, die den bei diesem Material anzunehmenden Winkel der inneren Reibung und der Kohäsion übersteigen. Solche großen Böschungswinkel, die durch Bohrungen ermittelt wurden, weisen ebenfalls auf eine Entstehung der abflußlosen Hohlformen durch austauendes Toteis bei rascher Verfüllung durch Schmelzwassersedimente hin. Die morphologische Gliederung einiger Toteislöcher in zwei bis drei kleinere Einsenkungen mit dazwischenliegenden Rücken läßt sich zwanglos durch den Zerfall eines größeren Eisblockes in einzelne Teilblöcke erklären.

3. Die Seitenmoränlandschaft ist am besten auf der Ostseite des Tales entwickelt. Während auf der Westseite lediglich eine Anhäufung von Geschieben, die meist solifluktil umgelagert sind, auf Reste der Seitenmoräne hindeutet, geht die Endmoränenstaffel auf der Ostseite in einen intakten Seitenmoränenkörper über.

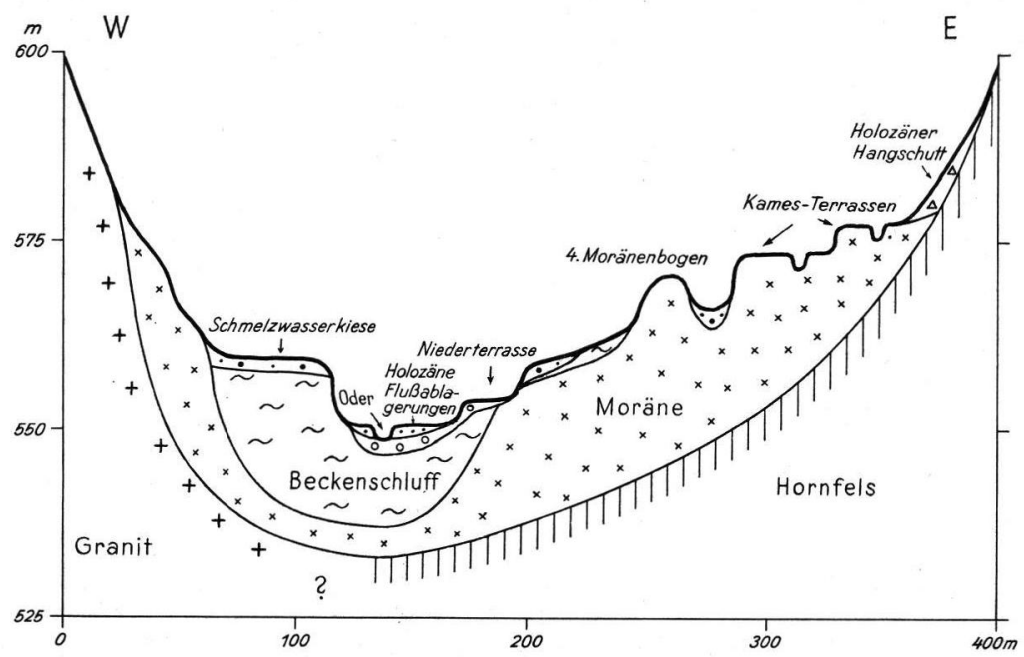

Abb. 3. Geologisches Querprofil durch das Zungenbecken des Odertalgletschers hinter der Moränenstaffel am Hahnenklee.

Die östliche Seitenmoräne ist glazifluviatil überprägt. Durch Schmelzwässer, die zwischen dem abtauenden Talgletscher und dem Talhang abflossen, wurde die Seitenmoräne kamesartig terrasiert, wobei zwei Terrassenflächen, die einen Höhenabstand von $3-5 \mathrm{~m}$ haben, entstanden. Auf den Terrassen liegt ein dünner Schleier von glazifluviatilen Kiesen und Sanden; an den Terrassenstufen kommt überall die Moräne zutage.

Durch zahlreiche Toteislöcher und Schmelzwasserrinnen erfährt die Seitenmoräne eine weitere morphologische Belebung. Die talabwärts verlaufende Sammelrinne wird von der 3-4 $\mathrm{m}$ tief unter das Niveau der unteren Kamesterrasse eingeschnittenen Hauptrinne, durch welche der erste Überlauf des von der Endmoräne abgedämmten Stausees erfolgte, gekappt.

$400 \mathrm{~m}$ weiter talaufwärts keilt der Seitenmoränenkörper aus bzw. löst sich in eine lose Reststreu von erratischen Geschieben auf, die bis $50 \mathrm{~m}$ hangaufwärts reicht. Damit ist ein Anhaltspunkt für die Rekonstruktion der Gletschermächtigkeit in diesem Talabschnitt gegeben. Als erratische Geschiebe kommen all die genannten Gesteine vor, die auch in der Grundmoräne enthalten sind, nicht nur Granitblöcke, die dort, wo sie auf 
Hornfels liegen, bereits von H. Poser \& J. Hövermann (1951) als erratische Geschiebe gedeutet, aber von H. LEMBKe (1961) aus anstehendem Granit, der durch Hornfels-Hangschutt verdeckt wird, abgeleitet werden.

4. Das Gletscherzungenbecken hinter der Moränenstaffel tritt morphologisch durch eine bis $200 \mathrm{~m}$ breite, von holozänen Hochflutsedimenten und periglaziär-solifluktilen bis -fluviatilen Kiesen der weichselkaltzeitlichen Niederterrasse eingenommene Ausweitung der Talsohle in Erscheinung. Die Mächtigkeit der Kiese beträgt 2-3 m.

Die fluviatil überprägte Talaue wird auf beiden Seiten von einer schmalen Terrassenleiste begleitet, deren Oberfläche 4-10 m über dem Bachbett liegt. Am markantesten ist die Terrassierung auf der Westseite des Tales. Dort wurde 1965 bei der Verbreiterung des Neumeierweges ein Anschnitt geschaffen, der einen Einblick in den geologischen Aufbau der Terrasse gestattet. Unter Solifluktionsschutt und glazifluviatilen Kiesen folgt eine Serie von Staubeckenablagerungen, die aus reinem Schluff im endmoränennahen Teil und aus einer Wechsellagerung von Kies, Sand und Schluff im Deltabereich des Stausees besteht.

Das der Endmoränenstaffel adäquate Gletscherzungenbecken, das nach dem Zurückschmelzen des Talgletschers dem von der Endmoräne abgedämmten Stausee Platz bot, wurde mit einem dichten Bohrnetz belegt, um Verbreitung, Mächtigkeit, Fazies- und Lagerungsverhältnisse der Staubeckenablagerungen zu ermitteln. Dabei wurde selbst noch unter den Niederterrassenkiesen bis $15 \mathrm{~m}$ mächtiger, z. T. warviger Beckenschluff erbohrt. Stückelt man die zahlreichen Bohrprofile zusammen, so ergibt sich eine maximale Schluffmächtigkeit von $23 \mathrm{~m}$. Aus den Lagebeziehungen des Schluffes zur Endmoräne wird ersichtlich, daß der Stausee fast randvoll mit Gletschertrübe angefüllt war, bevor er schließlich auf der Ostseite des Tales den abdämmenden Endmoränenwall durchbrach und sich über die in Abb. 2 dargestellte Hauptrinne einen Auslaß suchte.

Das Nordufer des Stausees ist auf der in Abb. 2 gezeigten Karte gerade noch erfaßt. Die von dem Gletscherbach eingeschütteten, grobklastischen Sedimente zeigen typische Deltaschichtung mit Einfallen bis zu $30^{\circ}$. An der Terrassenstufe kann das Auskeilen bzw. der Übergang der Deltasedimente in die Moräne, die hier aus dem Liegenden auftaucht und fortan talaufwärts den Terrassenkörper aufbaut, beobachtet werden. In der Talaue, die im Bereich des Stausees von einer ziemlich ebenen, periglaziär-fluviatilen Akkumulationsfläche gebildet wird, paust sich die zutagekommende Moräne in Gestalt von wallförmigen Blockanreicherungen und von unruhigem Buckelrelief durch.

W

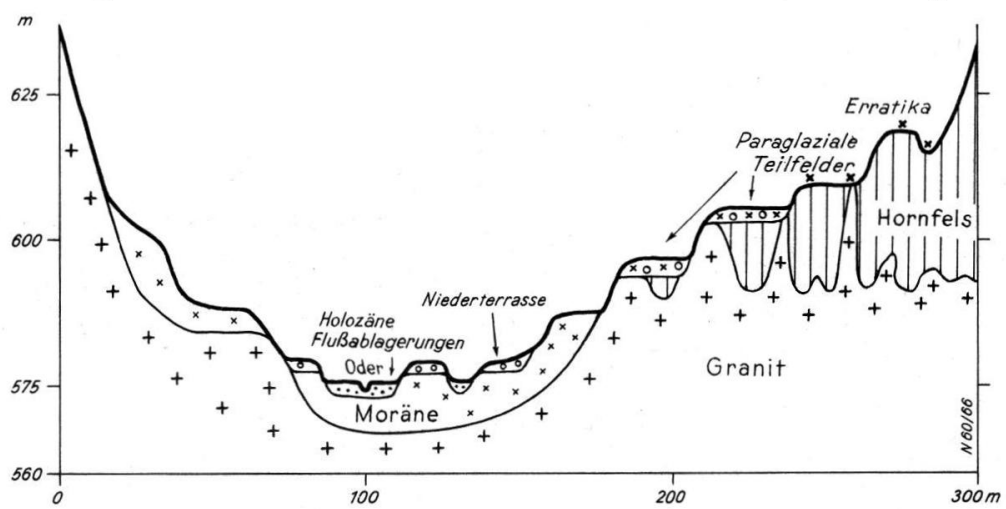

Abb. 4. Geologisches Querprofil durch das Odertal oberhalb des Gletscherzungenbeckens an der Schneise zum Pt. 735,2. 
Das steile Abtauchen der Moränenoberfläche unter die Stauseeablagerungen im Gletscherzungenbecken ist sowohl im Deltabereich als auch hinter der Endmoränenstaffel durch zahlreiche Bohrungen belegt. Aus den Bohrprofilen kann im Zusammenhang mit der Kartierung auf eine exarative Übertiefung des Gletscherzungenbeckens um mehrere Meter geschlossen werden. Zur weiteren Prüfung dieser Frage sind seismische Untersuchungen vorgesehen.

\section{Schlußbemerkungen}

Vorstehend wurde nur ein Teilabschnitt, nämlich das Kernstück des gesamten Untersuchungsgebietes, in dem es zunächst die Alternativfrage: pleistozäne Harzvergletscherung oder nicht - zu klären galt, zusammenfassend beschrieben. Nach den mitgeteilten Ergebnissen steht für mich außer Zweifel, daß der zentrale Oberharz im Pleistozän vergletschert war. Nach den neuen Befunden gibt es m. E. kein „Problem der pleistozänen Eigenvergletscherung des Harzes " mehr. Vielmehr haben sich die Probleme auf geologische, geomorphologische und paläoklimatische Spezialfragen, namentlich auf die Fragen nach dem geologischen Alter, dem Typ und nach dem Umfang der Vergletscherung bzw. der Vergletscherungen, verlagert. Abschließend sei kurz auf einige Ergebnisse zu diesem Fragenkreis eingegangen:

Im Odertal gibt es mehrere Moränenstaffeln, die als Rückzugslagen gedeutet werden. Talabwärts gehen die glaziären Ablagerungen in fluviatile Ablagerungen einer Terrasse über, deren sporadische Reste sich $2-4 \mathrm{~m}$ über das Niveau des Talbodens, der vorherrschend von der Niederterrasse eingenommen wird, erheben. Nach H. Poser \& J. HöverMANN (1951) soll dieser Übergang oberhalb des Oderhauses bei $435 \mathrm{~m}$ ü. N.N. liegen; diese Zahl kann aber m. E. nur als Annäherungswert beurteilt werden, denn ohne tiefere Bohrungen läßt sich die äußerste Eisrandlage bei der starken fluviatilen Überprägung der am weitesten talabwärts gelegenen Moränenreste nicht genau fixieren.

Die mit der Moräne korrespondierende Terrasse wurde von H. Poser \& J. HöverMANN (1951) als Obere Niederterrasse bezeichnet und der Weichseleiszeit zugeordnet. Ich schließe mich dieser stratigraphischen Einstufung aus Gründen, die in einer weiteren $\mathrm{Pu}-$ blikation erörtert werden sollen, an, gebe jedoch zu bedenken, daß diese Terrasse nicht mit einer der sicher datierten Terrassen des südlichen Harzvorlandes korreliert werden kann.

Die Eisdicke des weichselkaltzeitlichen Odertalgletschers, die nach der Höhenlage von paraglazialen Formen und erratischen Geschieben am Hang des Hahnenklee ca. $50 \mathrm{~m}$ betrug (s.o.), stieg im Firnbecken auf mindestens $80 \mathrm{~m}$ an, denn von dort erfolgte eine Transfluenz über den Sonnenberg-Paß ins Siebertal. Auch die nach W und N exponierten Täler des zentralen Oberharzes waren in der letzten Eiszeit, noch mehr jedoch in der Saale-Eiszeit, vergletschert. Die Untersuchungen hierüber sind z. Zt. noch im Gange, doch läßt sich jetzt schon absehen, daß wir im Harz mit zwei, vielleicht sogar mit drei Vereisungen rechnen müssen und daß wir bei den weiteren Arbeiten im zentralen Bereich vom Denkmodell umfangreicher Plateauvergletscherungen ausgehen müssen. Es gibt mehrere Indikationen für die Vorstellung, daß das mächtige Plateau-Eis eine ziemlich inaktive Masse war, die über längere Zeiträume der pleistozänen Kaltzeiten hinweg die Hochfläche zwischen Bruchberg und Brocken verhüllte, und daß deren Zentrum im Oderbecken lag, wo es eine reliefverhüllende Eiskuppel bildete, von der nur gelegentlich einzelne Zungen in die Täler hinabstießen. 


\section{Literaturverzeichnis}

Bode, A.: Die Moränenlandschaft im Odertale bei St. Andreasberg. Jb. k. pr. geol. L.-A. f. 1905, 26, 126-139, Berlin 1908.

BüDEL, J.: Die Gliederung der Würmkaltzeit. Würzb. geogr. Arb. 8, Würzburg 1960.

DuphorN, K.: Glaziäre, glazifluviatile und glazilimnische Ablagerungen und Formen im Odertal (Harz). Eiszeitalt. u. Gegenw. 17, 207, O'hringen 1966 (Vortragsbericht).

Kayser, E.: Zur Frage der Vergletscherung des Brockengebietes. Jb. k. pr. geol. L.-A. f. 1890, 11, 108-117, Berlin 1892.

Lembкe, H.: Glazial, Periglazial und die eiszeitliche Schneegrenze im Harz. Geologie, Jg. 10, H. 4/5, 442-460, Berlin 1961.

Marnzer, J.: Diluvialmorphologische Probleme des Harzes mit besonderer Berücksichtigung der Vergletscherungsfrage. Würzburg 1932.

Möвus, G.: Abriß der Geologie des Harzes. Leipzig 1966.

MüCKE, E.: Zur Frage der würmeiszeitlichen Brockenvergletscherung. Hercynia, N. F. 1, H. 4, 420-442, Leipzig 1964.

Poser, H. \& HövermanN, J.: Untersuchungen zur pleistozänen Harz-Vergletscherung. Abh. braunschw. wiss. Ges. 3, 61-115, Braunschweig 1951.

Reichelt, G.: Zur Frage pleistozäner Vergletscherung im Harz und Thüringer Wald. Erdk. 18, H. 1, 62-65, Bonn 1964.

WoldstedT, P.: Geologisch-morphologische Ubersichskarte des norddeutschen Vereisungsgebietes 1:1500 000. Berlin 1935. - - Bemerkungen zu meiner Geologisch-morphologischen Übersichtskarte des norddeutschen Vereisungsgebietes. Z. Ges. Erdk. Berlin, 282-295, Berlin 1935.

ZimmermanN, K. H.: Über Glescherspuren im Harz. N. Jb. Miner. usw., 155-159, Stuttgart 1868.

Manuskr. eingeg. 21. 5. 1968.

Anschrift des Verf.: Dr. K. Duphorn, Niedersächs. Landesamt für Bodenforschung, 3 HannoverBuchholz, Alfred-Bentz-Haus. 